molecules and theoretical values of angles and bond moments. Details will be published elsewhere.

Indian Institute of Science, S. K. Kulkarin Jatrar.

Malleswaram P.O., Bangalore.

Dec. 26.

INATURE, 153, 222 (1944).

\section{Solubilization of Dyes in Non-aqueous Solvents}

Tнотян a large number of instances of solubilization of water-insoluble substances like dyes, hydrocarbons, etc., in aqueous solutions of detergents are $k_{n o w n}{ }^{1}$, very few definite cases of the same phenomena have been reported for non-aqueous systems. In a recent note, $\mathrm{McBain}^{2}$, for the first time, has adduced qualitative evidence to show the existence of such solubilization by hydrocarbon-soluble soaps and detergents, and has very recently cited a few more instances ${ }^{3}$.

I have, however, observed that such solubilization is of rather frequent occurrence in the behaviour of resins dissolved in non-aqueous solvents. A systematic study has been undertaken and the results will be reported later. The present note records the hitherto unnoticed strong solubilizing power of resins, and also to direct attention to some peculiarities in this process not noticed by McBain and co-workers'. Their "experimental procedure has been to take approximately $1 \%$ solutions of pure or commercial detergents as supplied by the makers and add solid dye. Solubilization is indicated by the almost immediate coloration of the liquid." There is, however, an underlying source of error in this process of indicating solubilization, owing to the fact that some dyes ordinarily regarded as insoluble dissolve in traces in hydrocarbons to give a colourless solution which develops colour in presence of the dissolved resin (solubilizer). A good example is rhodamine; this dissolves slightly in benzene or toluene to give a colourless solution, which becomes pink on dissolving even a fraction of one per cent resin in it. So it is always necessary to check against such 'false' solubilization by noting the effect of adding the solubilizer to the dye-saturated solvent. This and other peculiar cases of chromatic interactions between dyes and resins have been observed and are receiving closer study.

A few cases of true solubilization are now being reported. The resins used have been crystalline abietic acid (the chief constituent of American rosin), purified glycerol triabietate (the chief constituent of ester gum) and soft resin of shellac, in 0.5 per cent solution in toluene and benzene at room temperature. Inerease of temperature has always been found to favour solubilization. So far, about fifty pure and commercial dyes have been tried, of which only about ten have shown true solubilization to a more or less extent as given in the following list, cases marked with an asterisk indicating a very strong effect : Ester gum solubilizes methyl violet*, phenoplastic blue $R B^{*}$, methylene blue, brilliant green, alkali blue, quinoline $A$, safranine, neutral violet and phenoplastic yellow $6 G$; abietic acid solubilizes monalite red $2 R S$, brilliant green, alkali blue and quinoline $A$; soft resin of shellac solubilizes methyl violet*, phenoplastic blue $R B^{*}$ and brilliant green.
McBain ${ }^{2}$ believes that non-aqueous solubilization, like its aqueous counterpart, is due to adsorption of the dyes on the micelles. We, however, find in this phenomena a manifestation of the already observed liquid-like behaviour of resins ${ }^{4}$, which might perhaps be ultimately due to the strong association tendency of the resin molecules, leading to formation of association dimers and polymers with the dye molecules.

A detailed examination of the mechanism of the process is, however, postponed until more data are forthcoming.

Indian Lac Research Institute,

S. R. Pality Namkum, Ranchi Dec. 14, 1942.

(Delayed in transit)

1 Pickering, J. Chem. Soc., 3, 86 (1917). Lester Smith, J. Phys. Chem; ; $\mathbf{3 6}, \mathbf{1 4 0 1}, \mathbf{1 6 7 2}, \mathbf{2 4 5 5}$ (1932). Hartley, "Wetting and Detergency"; 153 (1937). McBain and Woo, J. Amer. Chem. Soc., 60, 223 (1938), etc.

${ }^{2}$ McBain, Merrill and Vinograd, J.Amer. Chem. Soc., 62, 2880 (1940).

${ }^{3}$ McBain and Merrill, Ind. Eng. Chem., 34, 915 (1942).

${ }^{4}$ Palit, J. Ind. Chem. Soc., 19, 253 (1942).

\section{Reported Asymmetric Synthesis of Santonin}

Some time ago ${ }^{1}$ Paranjape, Phalnikar, Bhide and Nargund reported a synthesis of santonin from optically inactive materials: they now ${ }^{2}$ claim that their product was active, being almost entirely the natural $(l-)$ isomer. They state that the activity originated in the methylation of a 2-formylcyclohexanone derivative, and that the crude methylation product from 2-formylcyclohexanone (I) itself had the large specific rotation of $-26 \cdot 2^{\circ}$ in chloroform. Although they did not isolate 2-methyl-2-formylcyclohexanone (II), they claim that an optically active derivative was prepared in a state of purity.

Such an asymmetric synthesis from inactive materials violates no fundamental law and might theoretically be expected to occur once in about $\left(10^{10}\right)^{20}$ trials.

Nevertheless, Paranjape et al. claim to have achieved the asymmetric synthesis repeatedly. As we were interested in the products for another reason, it seemed worth while to repeat one of these experiments.

Sen and Mondal ${ }^{3}$ prepared (II), though not in a pure condition, and established its structure by hydrolysis to 2-methylcyclohexanone. We find that (II) may readily be purified by fractionation at low pressure, forming a colourless oil of camphoraceous odour, b.p. $47^{\circ} / 0.05 \mathrm{~mm} ., n_{D}^{18} 1.4683$ (Found: C, $68 \cdot 2,68 \cdot 4 ; \mathrm{H}, 8 \cdot 6,8 \cdot 4 . \mathrm{C}_{8} \mathrm{H}_{12} \mathrm{O}_{2}$ requires $\mathrm{C}, 68 \cdot 6$; $\mathrm{H}, 8.6$ per cent); the hydrolysis to methylcyclohexanone was confirmed. Neither the crude methylation product from (I) nor pure (II) showed any optical activity whatsoever.

J. W. CORNFORTH.

R. H. Cornforth.

M. J. S. Dewar.

Dyson Perrins Laboratory, Oxford.

1 Current Science, 12, 150 (1943).

2 NATURE, 153, 141 (1944).

3 . Ind. Chem. Soc., 5, 609 (1928). 